https://doi.org/10.23913/ciba.v8i16.91

Artículos Científicos

\title{
Estudio preliminar del fertirriego en el cultivo de la sandía (Citrullus lanatus, thumb) en Tolimán, Jalisco
}

\author{
Preliminary Study of the Fertirriego in the Culture of the Watermelon \\ (Citrullus Lanatus, Thumb) in Tolimán, Jalisco
}

Estudo preliminar da fertirrigação no cultivo de melancia (Citrullus lanatus, thumb) em Tolimán, Jalisco

J. de J. Sandoval Legazpi

Universidad de Guadalajara, Centro Universitario de la Costa Sur, Jalisco, México

slegazpi@cucsur.udg.mx https://orcid.org/0000-0003-4096-4042

O. E. Galindo Verdugo

Universidad de Guadalajara, Centro Universitario de la Costa Sur, Jalisco, México osvaldo-w@hotmail.com https://orcid.org/0000-0002-3251-8782

A. de J. Arellano Panduro

Universidad de Guadalajara, Centro Universitario de la Costa Sur, Jalisco, México aracelia@cucsur.udg.mx https://orcid.org/0000-0003-4598-0957

Lucio Ancira Sánchez

Universidad de Guadalajara, Centro Universitario de la Costa Sur, Jalisco, México lucio.ancira@cucsur.udg.mx https://orcid.org/0000-0003-1061-2310 


\section{Resumen}

El cultivo de la sandía (Citrullus lanatus, thumb) es de los que más requiere del factor hídrico, por lo que la implementación de una adecuada nutrición, aunado al uso eficiente del agua, puede ser una buena alternativa de manejo para los productores de este fruto en la región de Tolimán, Jalisco, y en otras regiones del país. Se realizaron muestreos de suelo y de agua para determinar su calidad para riego, así como del estado nutrimental del cultivo de la sandía mediante las metodologías del índice de balance de Kenworthy (IBK) y la desviación del óptimo porcentual (DOP). Los resultados muestran que el IBK es útil para los casos de muestras pequeñas y en estudios a nivel local (por valles y/o municipios). Asimismo, este estudio preliminar sugiere que el número de observaciones sea cuando menos de 48, porque así se tendrán tres rangos nutrimentales: alto, medio y bajo. Bajo este esquema se repartirían las observaciones en una población normal: 48 / $3=16$ observaciones por rango, lo que es suficiente para determinar los valores reales, sin tener que hacer uso de la desviación estándar.

Palabras clave: dosis, fertilizante, fertirriego, sandía.

\section{Abstract}

The cultivation of watermelon (Citrullus lanatus, thumb) is one that most requires the water factor, so the implementation of adequate nutrition, coupled with the efficient use of water, can be a good alternative management for watermelon producers from the Tolimán, Jalisco region, and from other regions of the country. Soil and water samples were taken to determine their quality for irrigation, as well as the nutrient status of the watermelon crop using the Kenworthy balance index (IBK) and the optimal percentage deviation (DOP) methodologies. The results show how the IBK is useful for small sample cases and in local studies (by valleys and/or municipalities). Likewise, this preliminary study suggests that the number of observations be at least 48 , because this way there will be three nutrimental ranges: high, medium and low. Under this scheme, the observations would be distributed in a normal population 48 / $3=16$ observations per range, enough to determine the real values, without having to make use of the standard deviation.

Keywords: dosage, fertilizer, fertigation, watermelon. 


\section{Resumo}

O cultivo de melancia (Citrullus lanatus, polegar) é o que mais requer o fator água, portanto a implementação de uma nutrição adequada, aliada ao uso eficiente da água, pode ser uma boa alternativa de manejo para os produtores deste tipo. frutas na região de Tolimán, Jalisco, e em outras regiões do país. Amostras de solo e água foram conduzidas para determinar a qualidade da irrigação, assim como o estado nutricional da cultura da melancia, utilizando as metodologias do Índice de Equilíbrio de Kenworthy (IBK) e o desvio percentual ótimo (PDO). Os resultados mostram que o IBK é útil para pequenos casos de amostra e em estudos no nível local (por vales e / ou municípios). Da mesma forma, este estudo preliminar sugere que o número de observações seja de pelo menos 48 , pois haverá três faixas nutricionais: alta, média e baixa. Sob este esquema, as observações seriam distribuídas em uma população normal: 48/3 = 16 observações por intervalo, o que é suficiente para determinar os valores reais, sem ter que fazer uso do desvio padrão.

Palavras-chave: dose, fertilizante, fertirrigação, melancia.

\section{Introducción}

El riego por goteo moderno tuvo sus orígenes en la zona árida de Israel. Ahí, el ingeniero israelí Simcha Blass, después de un periodo extenso de investigación de riego por goteo, en el año de 1966, crea el primer emisor para riego por goteo. En 1969, el emisor es patentado en Estados Unidos de América por Ischajahu Blass y Simcha Blass. El concepto de riego por goteo superficial se extendió en los 60 de Israel a Australia, Norteamérica y Sudáfrica y eventualmente a todo el mundo. Durante el periodo de 1981-2000, la microirrigación se incrementó de 0.4 millones a 3.2 millones de hectáreas. Estados Unidos fue el primer país en implementar esta tecnología con 1050000 hectáreas; México ocupó el octavo lugar mundial con 105000 hectáreas (Lamm, Ayars y Nakayama, 2007).

La sandía es una de las hortalizas más importantes para México debido a los altos volúmenes que se exportan. Dichas exportaciones alcanzaron los 283 millones de pesos en su acumulado de julio de 2016, lo que convierte a este fruto en uno de los productos más comercializados por México hacia el mundo (Secretaría de Agricultura y Desarrollo Rural 
Revista Iberoamericana de las Ciencias Biológicas y Agropecuarias

[Sagarpa]-Servicio de Información Agroalimentaria y Pesquera [SIAP], 2016). Asimismo, esta misma fuente señala que entre 2015 y 2016 las exportaciones de esta cucúrbita aumentaron en términos de valor 41 millones de dólares: se alcanzó una producción en el país de 753817 toneladas, producidas principalmente en diez estados de la República Mexicana. De entre esta decena de entidades, Jalisco ocupó el segundo lugar a nivel nacional, solo detrás del estado de Sonora, que aportó 23 \% de la producción nacional.

Para el ciclo otoño-invierno del 2018, la intención de siembra de sandía fue de 788 246 toneladas, lo que de cierta manera mantuvo una buena cantidad para exportación. Dicha producción se establecería en 23785 ha, con un promedio de 33.14 ton/ha (SIAP, 2018).

En lo que respecta a la región de estudio, donde se observa una baja producción de sandía, Sepúlveda (2009) señala que este bajo rendimiento se debe a tres principales factores limitantes de la producción: la fertilización, el racionamiento del agua y la enfermedad del damping off (marchitamiento fúngico temprano causado por invasión de hongo hospedero antes del surgimiento de la plántula). Dicha producción para el municipio de Tolimán fue de 8000 ton con una derrama económica de 11200000 pesos y para Tuxcacuesco fue de 9900 ton con una ganancia de 17820000 pesos.

Dado que esta zona de Jalisco es netamente agrícola y pecuaria, la derrama económica de este cultivo significa un gran arraigo de los lugareños por su región, ya que el cultivo de la sandía requiere de un número de jornales significativos. Además, de manera indirecta, existe una entrada extra a los pobladores por el transporte, movimiento de maquinaria agrícola, compra de insumos y consumo de combustible automotriz.

\section{Antecedentes}

Se entiende por fertirrigación a la aplicación de fertilizantes disueltos en el agua de riego, de una forma continua o intermitente. La fertirrigación no se puede hacer con todos los sistemas de riego, ya que la principal exigencia es obtener la máxima uniformidad en la distribución de los fertilizantes. Por eso, esta práctica se asocia básicamente a los sistemas de riego localizado de alta frecuencia, esto es, a los de riego por goteo y de microaspersión, aunque también puede aplicarse a través de riego por aspersión (Fuentes, 2003).

Este mismo autor, Fuentes (2003), señala que para lograr niveles altos de productividad en los cultivos se requiere de la aplicación externa de fertilizantes. Esta aplicación se debe realizar considerando las extracciones de nutrientes que requiere cada 
Revista Iberoamericana de las Ciencias Biológicas y Agropecuarias

cultivo, tomando en cuenta las características físico-químicas del suelo, el manejo del cultivo y el comportamiento fenológico de la especie. El análisis foliar es una herramienta importante para conocer el estado nutrimental de cultivos comerciales (Marín y Pérez de Roberti, 1992). De hecho, Marín y Pérez de Roberti (1992) señalan también que el análisis foliar pueden interpretarse por diferentes metodologías matemáticas o por comparación cualitativa de estos análisis y también por la respuesta de los cultivos a los fertilizantes.

Las metodologías más usadas, de acuerdo con Schulte and Kelling (2010), Cadahía (2005) y Campbell y Plank (2000), son las siguientes:

a) Niveles críticos (NC). Para la mayoría de los propósitos de diagnóstico, los análisis de plantas son interpretados con base en un "nivel crítico" para cada uno de los nutrientes, el cual es definido como "el contenido de un elemento en cierto tejido indicador por debajo del cual se espera una respuesta significativa a la aplicación de este elemento" (Howeler, 1993:6). Para muchos nutrientes, el rendimiento decrece justo antes de que se observe en forma visual un síntoma de deficiencia. Para algunos investigadores, el nivel crítico es la concentración del nutriente a $90 \%$ o $95 \%$ del máximo rendimiento. Este nivel crítico es definido para cada especie y en una etapa específica de crecimiento o madurez. Los intervalos de los nutrientes en algunos casos se representan como deficiente, bajo, suficiente, alto y excesivo.

b) Rangos de suficiencia (RS). En general, cuando un nutriente es deficiente, al agregar este resulta en un incremento en el crecimiento del cultivo y usualmente una mayor concentración del elemento en la planta y también causa un mejor rendimiento. No siempre el añadir fertilizante al suelo es garantía de que el cultivo se beneficiará, ya que pudiera no quedar disponible para la planta, es por eso por lo que los fisiólogos han hecho estudios sobre interacciones de nutrientes y así, después de varios análisis de plantas, descubrieron varias entre algunos elementos.

c) Índice de Kenworthy (IK). También se ha propuesto para interpretar el análisis foliar el índice de balance Kenworthy (IBK), por medio del cual se define el valor estándar como la media de la concentración nutrimental de hojas muestreadas en un estado particular de árboles de una especie vegetal con un desarrollo hortícola deseable. Para generar dicho valor estándar, se selecciona $10 \%$ o más de una población que exhiba un atributo deseado (por ejemplo, rendimientos elevados) y un coeficiente de variación (CV) menor de $34 \%$. Las concentraciones nutrimentales en una muestra se expresan como porcentaje del valor 
Revista Iberoamericana de las Ciencias Biológicas y Agropecuarias

estándar y se ajustan mediante el CV del estándar (Salazar y Lazcano, 1999; Maldonado et al. 2008).

d) Desviación del óptimo porcentual (DOP). Este índice de interpretación del análisis foliar permite, además del diagnóstico realista de una situación nutricional dada, conocer el orden de limitación tanto por exceso como por déficit de cada uno de los nutrientes considerados. Para la interpretación de los datos analíticos y para poder realizar el diagnóstico correcto de una situación nutricional dada, siempre es indispensable disponer de unos valores de referencia que pueden ser los óptimos nutricionales (punto medio del intervalo que coincide con la máxima cosecha). El índice de DOP es definido como la desviación porcentual de la concentración de un elemento con respecto a la concentración óptima considerada valor de referencia. El signo de la DOP para un determinado elemento será negativo en caso de déficit y positivo en caso de exceso. Cuando el contenido de la muestra coincide con el óptimo de referencia, será igual a cero (Montañés et al. 1990; Montañés et al. 1993).

Aunado a lo anterior, la DOP también es definida como un método estadístico que compara la concentración del nutriente en la muestra respecto a la norma, la cual se basa en un nivel nutrimental óptimo en el cual el cultivo expresa su máximo rendimiento potencial. El método DOP cuantifica el valor en que un nutriente se desvía con respecto a la norma individual. Una situación nutrimental óptima, para cualquier elemento, es definida por el índice de DOP igual a cero, lo que da el orden de limitación tanto por exceso como por déficit de cada uno de los nutrientes bajo estudio (Ventura et al., 2012).

e) Diagnosis de la composición de nutrientes (CND). Las relaciones correspondientes de los rendimientos más altos de la población (normas) son comparadas con la relación presente en el muestreo que está siendo analizado; una relación de concentración por sí misma no puede ser usada para diagnosticar problemas en la planta, pero combinaciones de relación de diferentes nutrientes pueden combinarse matemáticamente para determinar qué nutrientes son más probables para limitar el rendimiento. Los resultados de tales cálculos son los índices del sistema integrado de diagnóstico y recomendación (DRIS).

f) DRIS. Esta metodología detecta diferencias en la disponibilidad de los nutrientes analizados y las expresa en forma relativa, su amplitud de la base de datos puede ser determinante de los resultados obtenidos. Asimismo, debe ser utilizada como una herramienta más en el diagnóstico, dentro de un contexto que considere el análisis del suelo 
Revista Iberoamericana de las Ciencias Biológicas y Agropecuarias

y demás datos de la población en evaluación (Landriscini y Galantini, 2018). Es un acercamiento para la interpretación de resultados de análisis de plantas que involucra cientos de análisis de muestreo de un cultivo específico. Los muestreos son divididos dentro de dos poblaciones con altos y bajos rendimientos. El resultado analítico de cada población es estudiado para determinar qué criterio puede ser usado para distinguir el rendimiento entre el bajo y alto rendimiento de la misma población. Así, a medida que estos arrojan relaciones de la concentración de nutrientes en la planta, ofrecen mejores resultados que las simples concentraciones solas.

Una vez determinados los índices DRIS para cada nutrimento, se calcula el índice de desbalance nutricional (IDN) y se suman todos los índices, independientemente del signo. El valor más grande indica mayor desbalance nutricional y, por lo tanto, se esperaría un menor rendimiento (Reis Junior and Monnerat, 2003; Bangroo et al., 2010; Schulte and Kelling, 2010).

Para la interpretación de estos índices deben tenerse en cuenta las siguientes normas generales: 1) El valor numérico absoluto indica la importancia o gravedad de la situación anómala. 2) Lógicamente, cuando el índice DOP sea cero, el elemento correspondiente se halla en óptima concentración.

También la DOP permite conocer directamente el orden relativo de limitación entre los elementos considerados con base en ajustar las necesidades de fertilización con los correspondientes índices de DOP. Pero además permite clasificar la situación de los nutrientes definiendo tres categorías: los limitantes por déficit, los limitantes por exceso y aquellos que están en un nivel óptimo. Respecto a los elementos que tengan un valor negativo, significa que el nivel de la nutrición está por debajo de la nutrición óptima y que, por lo tanto, será necesario aumentar el programa de fertilización correspondiente a ese elemento. Los valores positivos indican que se deben reducir las cantidades del elemento en la fertilización.

La suma absoluta de los índices de DOP pueden ser usados como una medida relativa del balance nutricional en estudios de los cultivos y se concluye que cuanto menor sea el índice de DOP más cerca del óptimo estará el conjunto de elementos considerados (Montañés et al., 1993; Ventura, 2012; Landriscini y Galantini, 2018). Con base a lo anterior se plantean los siguientes objetivos. 


\section{Objetivos}

\section{General}

Obtener una dosis de fertilizante que se relacione con el análisis foliar para ser utilizada en riego por goteo en el cultivo de la sandía en el municipio de Tolimán, Jalisco.

\section{Específicos}

a) Determinar en el laboratorio los análisis de suelo, de la calidad del agua y los análisis foliares de las parcelas con sandía en Tolimán, Jalisco.

b) Conocer el estado nutrimental del cultivo de la sandía en Tolimán, Jalisco.

\section{Materiales y métodos}

\section{Descripción y ubicación del área de estudio}

El municipio de Tolimán se localiza entre las coordenadas $19^{0} 24^{\prime} 00^{*} \mathrm{~N}$ y $103^{0} 44^{\prime}$ 00“ (figura 1) y se encuentra a 760 m s. n. m. (Instituto Nacional de Estadística y Geografía [Inegi], 1981). Su población es 10310 habitantes (Inegi, 2015). El clima de Tolimán es BS1(h')w(i'), lo que significa que es un clima cálido seco en el invierno y con poca oscilación en la temperatura, con una precipitación pluvial de 570 mm (García, 1988). Los suelos pertenecen a la edad del cenozoico o terciario y las rocas son calizas, ígneas extrusivas, como riolitas, andesitas, basalto, tobas y brechas volcánicas. Las rocas sedimentarias son areniscas, conglomerados y depósitos aluviales (Inegi, 1981). 
Figura 1. Ubicación del municipio de Tolimán, Jalisco

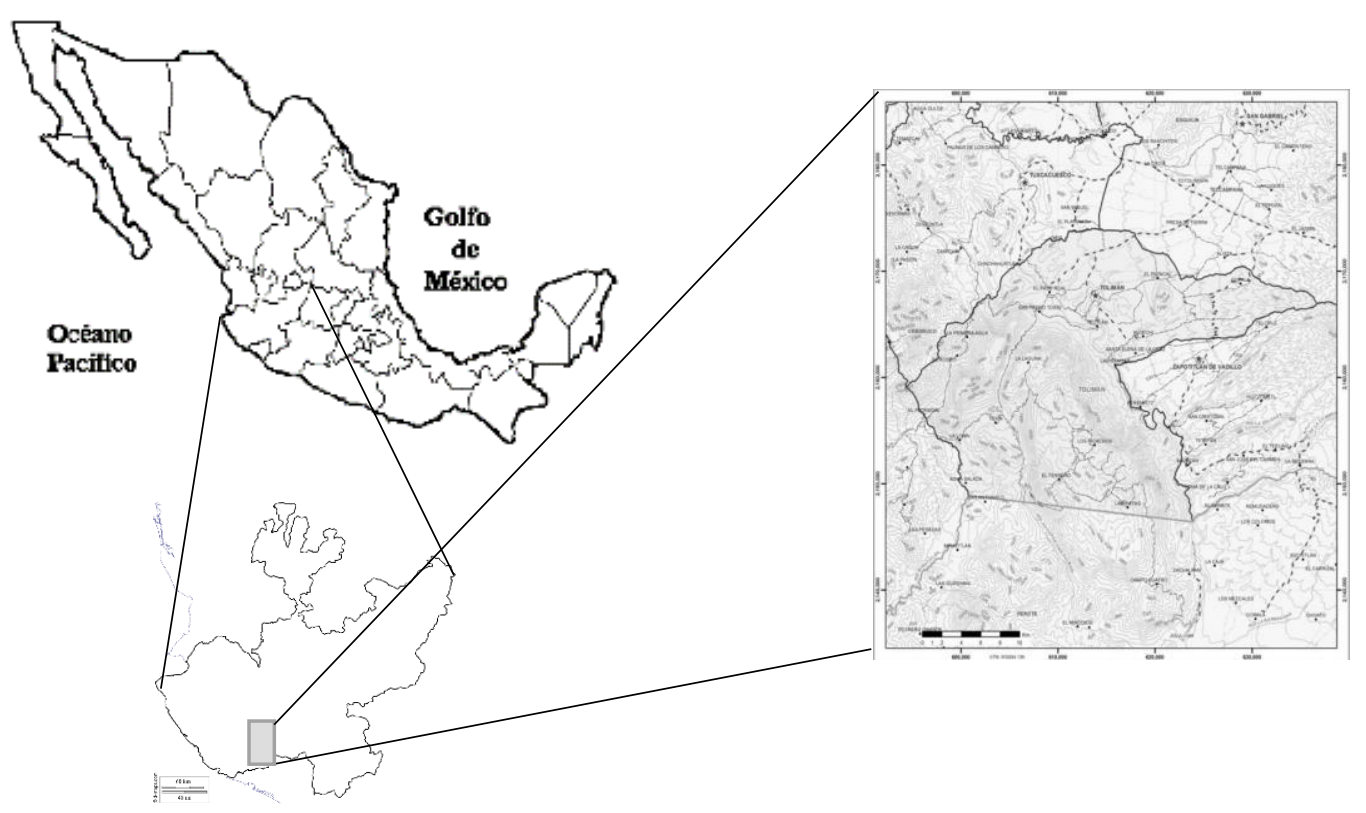

Fuente: Elaboración propia

La altura de la planicie antes de bajar al río Ayuquila-Armería es de $894 \mathrm{~m}$ y los pueblos del municipio de Tolimán donde se siembra sandía son el Paso Real y San Pedro Toxin, que están a una altura de $710 \mathrm{~m} \mathrm{s.} \mathrm{n.} \mathrm{m.} \mathrm{y} \mathrm{el} \mathrm{río} \mathrm{está} \mathrm{a} \mathrm{una} \mathrm{altura} \mathrm{sobre} \mathrm{el} \mathrm{nivel} \mathrm{del} \mathrm{mar}$ de 684 metros (la altura se determinó con un GPS 12 XL marca Garmin).

De acuerdo con el Instituto Nacional de Estadística, Geografía e Informática [INEGI] (1976) y la Organización de las Naciones Unidas para la Alimentación y la Agricultura [FAO] (2006), las seis parcelas en estudio se ubican en tres tipos de suelo: regosol calcárico, castañozem háplico y feozem calcárico. El tipo de suelo regosol cae en la clase 4, que son suelos minerales influenciados por su topografía y fisiografía. Estos pertenecen a terrenos bajos asociados a inundaciones recurrentes o humedad prolongada y en terrenos elevados o accidentados donde la formación del suelo ha sido impedida por erosión. Para el caso particular del regosol, este ocurre en material no consolidado y que solamente tiene un perfil superficial desarrollado. En la clase 8 se encuentran suelos de estepa entre los climas secos y la zona húmeda templada. Esta zona de transición tiene una vegetación de pastos y bosque seco. Dichas localidades tienen un proceso de lixiviado. Los castañozem se ubican en las partes menos profundas, con superficie cafesosa y acumulación de carbonato o yeso; surgen en la parte más seca de la estepa. Los feozem son suelos obscuros rojizos de regiones de pradera con alta base de saturación, pero no con signos visibles de acumulación de carbonato. 


\section{Preparación y muestreo del suelo}

Se tomaron las muestras de suelo a una profundidad de entre 0 y $40 \mathrm{~cm}$, tratando de considerar la parte radical de la sandía. Las mismas fueron enviadas a analizar al Laboratorio de Usos Múltiples del Centro Universitario de la Costa Sur de la Universidad de Guadalajara.

Una vez realizado el muestreo de suelos, se aró la parcela y se dejó 10 días bajo esta condición. Posteriormente, se realizaron dos pasos de rastra y se dejó descansar durante cuatro días. A continuación, se instalaron las camas de siembra de 2 metros de ancho, se acolcharon y se acondicionaron para el riego. Se sembró inmediatamente a una distancia de un metro entre planta y planta. Ese mismo día se instaló el abrigo meteorológico en la parcela del agricultor.

\section{Muestreo del agua para riego}

Se realizaron muestreos de agua con la finalidad de analizar su calidad para riego. Estos fueron realizados en el río Ayuquila-Armería, uno de los principales afluentes de la zona, así como en la zona de manantiales conocida como el acuífero Autlán, fuentes de agua utilizadas para riego por los productores de sandía y otros cultivos para este y otros municipios.

\section{Fertilización}

El fertirriego se realizó mediante un tensiómetro y con una lectura de 13 cb, es decir, para evitar que el suelo estuviera saturado. Las tomas de lectura se realizaron de manera diaria. Un tensiómetro estuvo a $30 \mathrm{~cm}$ de profundidad y a $30 \mathrm{~cm}$ de distancia del cuello de la planta. Este sirvió para regar el cultivo cuando este tenía de 1 día a 20 días después del trasplante (ddt); y otro tensiómetro se colocó a $50 \mathrm{~cm}$ de profundidad, que sirvió para regar la planta cuando empezó a soltar guía.

La solución nutritiva se preparó en tambos con 150 lts. Se pusieron todos los fertilizantes menos el nitrato de calcio que iba en otro tambo y se aplicó media hora después de que se aplicó la primera solución.

En cada riego se acidificó la solución nutritiva con ácido sulfúrico a 97 \%; y se dejó la solución a un $\mathrm{pH}$ de 5.5. Los fertirriegos se hicieron de acuerdo con la tabla 1, mientras que el manejo de la sanidad de la planta de acuerdo con la tabla 2. 
Tabla 1. Fertirriego en $\mathrm{kg} / \mathrm{ha} / \mathrm{riego}$

\begin{tabular}{|c|c|c|c|c|c|c|}
\hline ddt & $\begin{array}{c}\text { N como } \\
\text { fosfonitrato }\end{array}$ & $\begin{array}{c}\mathrm{P}_{2} \mathrm{O}_{5} \text { como fosfato } \\
\text { monoamónico }\end{array}$ & $\begin{array}{c}\mathrm{K}_{2} \mathrm{O} \text { como } \\
\mathrm{KNO}_{3}\end{array}$ & $\begin{array}{c}\text { Ca como } \\
\mathrm{CaNO}_{3}\end{array}$ & $\begin{array}{c}\text { Mg como } \\
\mathrm{MgSO}_{4}\end{array}$ & $\begin{array}{l}\mathrm{S} \mathrm{como} \\
\mathrm{MgSO}_{4}\end{array}$ \\
\hline $\begin{array}{l}0-15 \\
4 \text { riegos }\end{array}$ & 11.360 & 11.880 & 16.300 & 7.500 & 12.500 & \multirow[t]{4}{*}{$\begin{array}{c}\mathrm{Al} \text { aplicar } \\
\mathrm{MgSO}_{4}\end{array}$} \\
\hline $\begin{array}{l}16-30 \\
8 \text { riegos }\end{array}$ & 3.787 & 3.960 & 8.150 & 3.750 & 6.250 & \\
\hline $\begin{array}{l}31-45 \\
16 \text { riegos }\end{array}$ & 1.893 & 1.980 & 6.112 & 2.812 & 3.125 & \\
\hline $\begin{array}{l}46-60 \\
16 \text { riegos }\end{array}$ & 2.840 & 2.970 & 6.112 & 2.812 & 3.125 & \\
\hline Total & 150 & 95 & 150 & 30 & 30 & 26 \\
\hline
\end{tabular}

Fuente: Elaboración propia

\section{Muestreo foliar}

$\mathrm{El}$ análisis foliar o de tejidos vegetales es una herramienta esencial para el diagnóstico nutricional de cultivos. Consiste en medir el contenido total de los nutrientes presentes en las hojas o en otra parte de la planta a través de procedimientos químicos específicos (Martínez y Soriano, 2014).

Para la toma de muestras se procuró no tomar las mismas sobre plantas situadas cerca de caminos, edificios, etc., ni en aquellas que tuvieran acumulación de residuos por algún tipo de tratamiento por aspersión, ya que se entendió que los análisis no serían representativos.

Para este estudio, además de lo anterior, el muestreo de hojas se hizo a los 50 ddt, tomando 12 hojas de la guía principal, la décima hoja del ápice al tallo, una hoja por planta. El muestreo del agua de riego se realizó mediante la determinación de dureza del agua, y se obtuvo principalmente presencia de nitratos, nitritos, cloro con el espectrofotómetro de Hach. Este muestreo se realizó tres veces durante tres meses; el análisis foliar y el análisis químico del agua se hicieron en el Laboratorio de Usos Múltiples del Centro Universitario de la Costa Sur de la Universidad de Guadalajara. 
Tabla 2. Enfermedad y/o plaga, producto y dosis a aplicar en $\mathrm{kg} / \mathrm{ha}$

\begin{tabular}{|l|l|l|}
\hline Enfermedad & Producto & Dosis kg/ha \\
\hline $\begin{array}{l}\text { Mildiu } \\
\text { Pseudoperonospora }\end{array}$ & Mancozeb 80 & $1.5 \mathrm{l}$ \\
Tattoo c & $2.0 \mathrm{lt}$ \\
\hline $\begin{array}{l}\text { Cenicilla polvorienta } \\
\text { Erysiphe } \\
\text { chicoracearum }\end{array}$ & Zineb micro 80 & $1.5 \mathrm{~kg}$ \\
\hline Nemátodos & Blindaje 50 & $0.5 \mathrm{~kg}$ \\
\hline Pulgón & & \\
& Nemacur & $4-81$ \\
\hline Aiabrótica & Aflix & 1 \\
& Actara & $0.4 \mathrm{~kg}$ \\
Thiodan & $1.5 \mathrm{lt}$ \\
& Methan & $1.5 \mathrm{lt}$ \\
\hline Mosquita blanca & Parathion metilico & $20.0 \mathrm{~kg}$ \\
& Confidor 350 & 1.01 \\
& Sevin $80 \%$ & $1.0 \mathrm{~kg}$ \\
\hline Minador de la hoja & Diazinon & $0.8 \mathrm{lt}$ \\
& Folimat & 1.01 \\
& Ambush & 0.41 \\
\hline Diazinon & 0.81 \\
& Lorsban 480 EM & 1.51 \\
& Endocoral 50 & 1.01 \\
\hline Aannate 90 & $0.4 \mathrm{~kg}$ \\
& Ambush 50 & $0.4 \mathrm{lt}$ \\
\hline
\end{tabular}

Fuente: Elaboración propia

\section{Estado nutrimental de la sandía}

Para determinar el estado nutrimental del cultivo de la sandía, se utilizó el IBK y la DOP. Los cálculos para el IBK fueron:

Si $X$ es > que el valor estándar:

$$
P=\frac{X}{X} 100 \quad I=(P-100)\left(\frac{C V}{100}\right) \quad B=P-I
$$

Si $X$ es < que el valor estándar:

$$
P=\frac{X}{X} 100 \quad I=(100-P)\left(\frac{C V}{100}\right) \quad B=P+I
$$


Donde:

$P=$ porcentaje del valor estándar.

$I=$ ajuste del valor estándar mediante el coeficiente de variación.

$X=$ concentración del nutriente de la muestra.

$C V=$ coeficiente de variación.

$\dot{X}=$ media de la población.

$B=$ índice de balance.

Además, con los IBK se elaboró el diagnóstico en solo tres rangos: bajo (83-93), medio (94-104) y alto (105-115), aunque algunos investigadores utilizan cinco rangos (Maldonado et al., 2008; Maldonado, Etchevers, Rodríguez y Colinas, 2001).

El índice de DOP se calculó con la siguiente fórmula:

$$
D O P=C \frac{100}{C r e f}-100
$$

Donde:

$C$ : Es la concentración foliar del elemento en la muestra analizada.

C ref.: Es el óptimo de referencia del mismo elemento, con las mismas características en que fue tomada la muestra problema.

El siguiente punto muestra los resultados generados con base a los objetivos planteados: 


\section{Resultados y discusión}

\section{Análisis de suelo}

Tabla 3. Análisis físico-químico de suelos antes de la siembra de los poblados Paso Real y San Pedro Toxin del municipio de Tolimán, Jalisco. Profundidad de 0-25 cm y $25-50 \mathrm{~cm}$, respectivamente

\begin{tabular}{|l|c|c|c|c|c|c|}
\hline & Parcela 1* & Parcela 2 & Parcela 3 & Parcela 4 & Parcela 5 & Parcela 6 \\
\hline Textura & F-A & F-A & A-F & F-A & A-F & A-F \\
\hline $\mathrm{pH}$ & 8.27 & 8.05 & 8.28 & 7.73 & 7.69 & 7.89 \\
\hline $\mathrm{CE}$ & 0.2 & 0.02 & 0.05 & 0.02 & 0.02 & 0.07 \\
\hline $\mathrm{MO}$ & 0.69 & 0.26 & 0.13 & 0.76 & 0.69 & 0.17 \\
\hline $\mathrm{P}$ & 13.45 & 12.71 & 12.65 & 13.67 & 13.47 & 12.26 \\
\hline $\mathrm{K}$ & 50.7 & 48 & 47.7 & 51.4 & 51.6 & 46.6 \\
\hline $\mathrm{Ca}$ & 480 & 476 & 461 & 506 & 506 & 453 \\
\hline $\mathrm{Mg}$ & 4571 & 4200 & 4631 & 3615 & 3701 & 3667 \\
\hline & 476 & 461 & 450 & 461 & 453 & 461 \\
\hline Textura & F-A & F-A & A-F & F-A & A-F & A-F \\
\hline $\mathrm{pH}$ & 8.13 & 8.30 & 8.28 & 7.53 & 7.82 & 7.66 \\
\hline $\mathrm{CE}$ & 0.2 & 0.1 & 0.08 & 0.02 & 0.01 & 0.08 \\
\hline $\mathrm{MO}$ & 0.07 & 0.07 & 0.07 & 0.58 & 0.47 & 0.07 \\
\hline $\mathrm{N}$ & 12.20 & 12.46 & 12.21 & 13.54 & 13.43 & 12.20 \\
\hline $\mathrm{P}$ & 46.6 & 47.4 & 46.3 & 51.3 & 51.9 & 47.0 \\
\hline $\mathrm{K}$ & 446 & 446 & 450 & 491 & 495 & 450 \\
\hline $\mathrm{Ca}$ & 4162 & 4646 & 4612 & 3506 & 4125 & 3611 \\
\hline $\mathrm{Mg}$ & 438 & 438 & 438 & 453 & 450 & 446 \\
\hline
\end{tabular}

F-A = Franco Arenoso; A-F = Areno Francoso; $N$ = kg/ha. Los demás elementos en ppm

*En la parcela uno se instaló la parcela demostrativa y también estaba el cultivo de un agricultor, por lo que aquí estaba la parcela uno y dos de los siguientes cuadros.

Fuente: Elaboración propia

La tabla 3 muestra lo siguientes resultados:

Para suelos cultivados de clima subtropical o templado, Castellanos, Uvalle y Aguilar (2000) clasifican los suelos de textura arenosa como normales si tienen una cantidad de materia orgánica (MO) entre $1.21 \%$ y $1.60 \%$ y si los suelos son francos deben contener 1.81-2.30 \% de MO para considerarlos normales, así que los suelos en estudio se clasifican como suelos muy pobres. 
Respecto al $\mathrm{pH}$ tres suelos variaron de 7.64 a 7.89, por lo que se pueden clasificar como ligeramente alcalinos; y otros tres suelos variaron de 8.05 a 8.28 , que son medianamente alcalinos (Troe y Thompson, 2005).

La conductividad eléctrica (CE) no es un problema de salinidad ni afecta el crecimiento debido a la presión osmótica de la solución del suelo, ya que esta se encuentra en una concentración menor a 2.0 mmhos por cm (Richards, 1977).

El $N$ del suelo en las 6 parcelas varió de 12.26 a $13.67 \mathrm{~kg} / \mathrm{ha}$, por lo que se pueden clasificar como suelos muy bajos, ya que Castellanos et al. (2000) señalan que un suelo medio en nitrógeno tiene de $101-150 \mathrm{~kg} / \mathrm{ha}$.

Los nutrientes $\mathrm{P}, \mathrm{K}, \mathrm{Ca}$ y $\mathrm{Mg}$ son clasificados, de acuerdo con Castellanos et al. (2000), como altos, moderadamente altos, altos y moderadamente altos, respectivamente (tabla 3). Los resultados anteriores son para la profundidad de $0-25 \mathrm{~cm}$; no se comenta la profundidad de 25 a $50 \mathrm{~cm}$ ya que matemáticamente existe una diferencia que no es significativa tanto en nutrientes como en las otras variables del suelo.

\section{Análisis del agua de riego}

El pH del río y el manantial varió de 7.4 a 8.6 (ver tabla 4). La Norma Oficial Mexicana de Ecología (1996) dice que se permiten entre 5 a 10 unidades de pH; aun cuando Ayers y Westcot (1994) comentan que se debe tener restricciones ligeras con $\mathrm{pH}$ por arriba de 8.4, porque se puede reducir la producción para algunos cultivos.

La CE del agua del río varió de 0.40 a 0.98 siemens por metro (SI) y la del manantial de 0.36 a 0.80 (tabla 4). Al respecto, Ayers y Westcot (1994) comentan que cuando la CE esté entre 0.7 y $3.0 \mathrm{mmhos} / \mathrm{cm}$ se deben tener restricciones ligeras, por lo cual en este estudio la $\mathrm{CE}$ se considera como normal. Estos mismos autores dicen que la alcalinidad medida como bicarbonatos $\left(\mathrm{HCO}_{3}\right)$ debe estar en un rango de 0 a 10 meq/l, por lo que, al dividir los mg/l de bicarbonatos entre 61.01, que es el peso molecular del bicarbonato, solo las parcelas que se regaron con el agua del manantial estuvieron por encima de lo permitido en el mes de enero.

El calcio varió de 12-103.2 y el Mg de 102 a 1415.2 mg/l (véase tabla 4). Al respecto, Motsara y Roy (2008) comentan que en el análisis del agua el Ca y el Mg no se comportan idénticamente en el sistema del suelo, ya que el $\mathrm{Mg}$ deteriora la estructura del suelo, particularmente en las aguas donde predomina el $\mathrm{Na}$ o donde las aguas son ligeramente 
salinas. Un alto nivel de $\mathrm{Mg}$ usualmente promueve un desarrollo más alto de sodio intercambiable en suelos irrigados. Es por eso por lo que, de acuerdo con las tres categorías basadas en la relación de $\mathrm{Mg} / \mathrm{Ca}$, las aguas son clasificadas como: $<1.5$ seguras; 1.5-3.0 moderadas, y > 3.0 peligrosas. Ayers y Westcot (1994) señalan que para el calcio y magnesio el rango permitido es de 20 y 5 meq/l, respectivamente (400 y 60 mg/l).

Rincón (2005) comenta que todas las aguas que lleven en disolución como mínimo $1.5 \mathrm{meq} / \mathrm{l} \mathrm{de} \mathrm{Ca}(30 \mathrm{mg} / \mathrm{l})$ y $1 \mathrm{meq} / \mathrm{l} \mathrm{de} \mathrm{Mg}(12.16 \mathrm{mg} / \mathrm{l})$ aportan suficiente Ca y $\mathrm{Mg}$ para los cultivos que no requieren de cantidades muy altas. No obstante, debido al elevado grado de lavado y a la deficiencia de Ca que presenta la sandía cuando se encuentra en la fase de desarrollo del fruto, es recomendable hacer aportaciones preventivas de $\mathrm{Ca}$ y $\mathrm{Mg}$ durante esta fase para evitar posibles deficiencias: las cantidades recomendables a reponer son de $50 \%$ de las extracciones totales durante el ciclo del cultivo.

La cantidad de cloruros varió de 86 a 109 mg/l en el río y en el manantial de 56 a 118 mg/l (tabla 4), por lo que este elemento no representa un daño para los cultivos de la región, ya que el criterio ecológico sobre la calidad del agua que tiene el Instituto Nacional de Ecología (1989) recomienda que los cloruros no deben ser mayores a la cantidad de 147.5 $\mathrm{mg} / \mathrm{l}$.

Respecto al elemento Na, Ayers y Westcot (1994) recomiendan que el agua no tiene ninguna restricción si el valor de este no excede el valor de $3 \mathrm{meq} / \mathrm{l}(69 \mathrm{mg} / \mathrm{l})$. Esto refuerza lo dicho en lo referente a la $\mathrm{CE}$, de que las sales no son dañinas para los cultivos de la zona del municipio de Tolimán (ver tabla 4). 
Tabla 4. Análisis del agua del río y del nacimiento durante el cultivo de la sandía

\begin{tabular}{|l|c|c|c|c|c|c|}
\hline & \multicolumn{2}{|c|}{$8 / \mathrm{I} / 2010$} & \multicolumn{2}{c|}{$3 / 11 / 2010$} & \multicolumn{2}{c|}{$5 / \mathrm{III} / 2010$} \\
\hline & Río & Manantial & Río & Manantial & Río & Manantial \\
\hline $\mathrm{pH}$ & 8.6 & 8.5 & 7.5 & 7.5 & 7.5 & 7.4 \\
\hline $\mathrm{CE}$ & 0.98 & 0.80 & 0.40 & 0.80 & 0.69 & 0.36 \\
\hline Alcalinidad $\left(\mathrm{HCO}_{3}\right)$ & 368.7 & 1425.9 & 305.5 & 323.9 & 103.1 & 200.9 \\
\hline $\mathrm{NO}_{3}$ & 16 & 16 & 14 & 15 & 15 & 14 \\
\hline $\mathrm{PO}_{4}$ & 0.06 & 0.02 & 0.06 & 0.02 & 0.06 & 0.02 \\
\hline $\mathrm{K}$ & 21 & 21 & 22 & 23 & 22 & 22 \\
\hline $\mathrm{Ca}$ & 12 & 84.8 & 103.2 & 151.2 & 92 & 50 \\
\hline $\mathrm{Mg}$ & 488 & 1415.2 & 272.8 & 270.8 & 102 & 190 \\
\hline $\mathrm{SO}$ & 14 & 15 & 16 & 13 & 17 & 12 \\
\hline $\mathrm{Cl}$ & 86.8 & 84 & 105 & 118 & 109 & 56 \\
\hline $\mathrm{Na}$ & 22 & 20 & 24 & 25 & 22 & 21 \\
\hline
\end{tabular}

Fuente: Elaboración propia

\section{Análisis foliar}

En la tabla 5, el IBK indica que en la parcela 4 y 7 el elemento que está restringiendo la producción es el $\mathrm{N}$, ya que este resulta en los análisis de suelo como muy bajo, y en la parcela 4 solo se aplicó $56 \mathrm{~kg}$ de N (tabla 6) y no fueron suficientes para nutrir a 46.6 ton/ha (tabla 7). En la parcela 7 solo se aplicaron $22 \mathrm{~kg}$ de N, por lo que provocó que este elemento limitara el rendimiento con una producción de tan solo 25 ton/ha.

En la parcela seis resultó que el fósforo es el principal limitante de la producción; lo confirma esta misma parcela, ya que es la que menos fósforo tiene en el suelo.

Asimismo, en la parcela tres el $\mathrm{K}$ fue el que limitó la producción y esto pudiera deberse a que cuanta más cantidad de K se necesita en el desarrollo del fruto, la profundidad del suelo es donde está el pH más alcalino y esto limita la absorción del mismo, ya que también se refleja en los datos de tabla 7; allí se observa que el tamaño promedio del fruto para exportación es de los más bajos en los rendimientos por arriba de los $30 \mathrm{~kg} / \mathrm{ha}$.

El Ca limita la producción en la parcela dos y esto pudiera ser debido a que en esta es en donde más fósforo se aplicó; y Foth (1990) comenta que cuando el pH del suelo está por arriba de 7.2, el anión fosfato reacciona con el Ca y forma el compuesto fosfato de calcio insoluble. 
El S es el elemento que más limita la producción en las parcelas uno y cinco. Mientras que la parcela 1, que es la parcela demostrativa, indica que si queremos sacar rendimientos por arriba de 45 toneladas se tiene que aplicar al suelo más de $26 \mathrm{~kg}$ de $\mathrm{S}$. Por otro lado, en la parcela cinco los suelos son muy pobres en MO. Al respecto, Thompson (1974) y Fassbender y Bornemisza (1987) señalan que existe una relación directa entre la cantidad de MO del suelo y la cantidad de azufre disponible para los cultivos. También es cierto que esta parcela tuvo un mal manejo del agua, por lo cual se dio el más bajo rendimiento con tan solo 18.9 ton/ha.

Tabla 5. Diagnóstico nutrimental para los siete sitios en el cultivo de la sandía en Tolimán, Jalisco, por los métodos IBK y DOP

\begin{tabular}{|c|c|c|c|c|c|c|c|c|}
\hline & & \multicolumn{7}{|c|}{ Composición Nutrimental de la hoja al inicio del fruto } \\
\hline & & $\mathbf{N}$ & $\mathbf{P}$ & $\mathbf{K}$ & $\mathbf{C a}$ & Mg & $\mathbf{S}$ & IBN \\
\hline \multirow[t]{2}{*}{1} & $\mathrm{CNF}$ & 3.9 & 0.41 & 3.9 & 1.9 & 0.67 & 0.51 & \\
\hline & IBK & 100 & 102 & 96 & 100 & 100 & 92 & 590 \\
\hline & ORN & \multicolumn{7}{|c|}{$\mathrm{S}>\mathrm{K}>\mathrm{N}=\mathrm{Ca}=\mathrm{Mg}>\mathrm{P}$} \\
\hline & DOP & +10 & 0 & +11 & -20 & +18 & +70 & 129 \\
\hline & ORN & \multicolumn{7}{|c|}{$\mathrm{Ca}>\mathrm{P}>\mathrm{N}>\mathrm{K}>\mathrm{Mg}>\mathrm{S}$} \\
\hline \multirow[t]{2}{*}{2} & $\mathrm{CNF}$ & 3.6 & 0.39 & 4.1 & 1.7 & 0.61 & 0.53 & \\
\hline & IBK & 93 & 98 & 101 & 90 & 92 & 95 & 569 \\
\hline & ORN & \multicolumn{7}{|c|}{$\mathrm{Ca}>\mathrm{Mg}>\mathrm{N}>\mathrm{S}>\mathrm{P}>\mathrm{K}$} \\
\hline & DOP & +2 & -5 & +17 & -29 & +7 & +77 & 137 \\
\hline & ORN & \multicolumn{7}{|c|}{$\mathrm{Ca}>\mathrm{P}>\mathrm{N}>\mathrm{Mg}>\mathrm{K}>\mathrm{S}$} \\
\hline \multirow[t]{5}{*}{3} & $\mathrm{CNF}$ & 4.1 & 0.44 & 3.9 & 1.9 & 0.71 & 0.65 & \\
\hline & IBK & 105 & 109 & 96 & 100 & 106 & 114 & 630 \\
\hline & ORN & \multicolumn{7}{|c|}{$\mathrm{K}>\mathrm{Ca}>\mathrm{N}>\mathrm{Mg}>\mathrm{P}>\mathrm{S}$} \\
\hline & DOP & +16 & +7 & +11 & -20 & +25 & +117 & 196 \\
\hline & ORN & \multicolumn{7}{|c|}{$\mathrm{Ca}>\mathrm{P}>\mathrm{K}>\mathrm{N}>\mathrm{Mg}>\mathrm{S}$} \\
\hline \multirow[t]{5}{*}{4} & $\mathrm{CNF}$ & 3.9 & 0.46 & 4.3 & 2.1 & 0.68 & 0.6 & \\
\hline & IBK & 100 & 114 & 105 & 110 & 101 & 106 & 636 \\
\hline & ORN & \multicolumn{7}{|c|}{$\mathrm{N}>\mathrm{Mg}>\mathrm{K}>\mathrm{S}>\mathrm{Ca}>\mathrm{P}$} \\
\hline & DOP & +10 & +12 & +23 & -12 & +19 & +100 & +176 \\
\hline & ORN & \multicolumn{7}{|c|}{$\mathrm{Ca}>\mathrm{N}>\mathrm{P}>\mathrm{Mg}>\mathrm{K}>\mathrm{S}$} \\
\hline 5 & $\mathrm{CNF}$ & 3.6 & 0.34 & 4.1 & 1.8 & 0.63 & 0.46 & \\
\hline & IBK & 93 & 86 & 101 & 95 & 94 & 84 & 553 \\
\hline
\end{tabular}


Revista lberoamericana de las Ciencias Biológicas

y Agropecuarias

\begin{tabular}{|c|c|c|c|c|c|c|c|c|}
\hline & ORN & \multicolumn{7}{|c|}{$\mathrm{S}>\mathrm{P}>\mathrm{N}>\mathrm{Mg}>\mathrm{Ca}>\mathrm{K}$} \\
\hline & DOP & +2 & -17 & +17 & -24 & +11 & +53 & 124 \\
\hline & ORN & \multicolumn{7}{|c|}{$\mathrm{Ca}>\mathrm{P}>\mathrm{N}>\mathrm{Mg}>\mathrm{K}>\mathrm{S}$} \\
\hline \multirow[t]{2}{*}{6} & $\mathrm{CNF}$ & 4.3 & 0.38 & 4 & 1.9 & 0.74 & 0.61 & \\
\hline & IBK & 110 & 95 & 98 & 100 & 110 & 108 & 621 \\
\hline & ORN & \multicolumn{7}{|c|}{$\mathrm{P}>\mathrm{K}>\mathrm{Ca}>\mathrm{S}>\mathrm{N}>\mathrm{Mg}$} \\
\hline & DOP & +21 & -7 & +14 & -20 & +30 & +103 & 195 \\
\hline & ORN & \multicolumn{7}{|c|}{$\mathrm{Ca}>\mathrm{P}>\mathrm{K}>\mathrm{N}>\mathrm{Mg}>\mathrm{S}$} \\
\hline 7 & $\mathrm{CNF}$ & 3.8 & 0.4 & 4.2 & 2 & 0.67 & 0.58 & \\
\hline & IBK & 98 & 100 & 103 & 105 & 100 & 103 & 609 \\
\hline & ORN & \multicolumn{7}{|c|}{$\mathrm{N}>\mathrm{P}>\mathrm{Mg}>\mathrm{K}>\mathrm{S}>\mathrm{Ca}$} \\
\hline & DOP & +8 & -2 & +20 & -16 & +18 & +93 & 157 \\
\hline & ORN & \multicolumn{7}{|c|}{$\mathrm{Ca}>\mathrm{P}>\mathrm{N}>\mathrm{Mg}>\mathrm{K}>\mathrm{S}$} \\
\hline
\end{tabular}

$\mathrm{IBN}=$ Índice de balance nutrimental. $\mathrm{CNF}=$ Concentración nutrimental foliar. ORN= Orden de requerimiento nutrimental.

Fuente: Elaboración propia

Tabla 6. Dosis de fertilizante aplicada durante el cultivo por medio de la fertirrigación en $\mathrm{kg} / \mathrm{ha}$

\begin{tabular}{|c|c|c|c|c|c|c|}
\hline $\begin{array}{c}\text { Núm. } \\
\text { de } \\
\text { parcela }\end{array}$ & $\mathbf{N}$ & $\mathbf{P}$ & $\mathbf{K}$ & $\mathbf{C a}$ & $\mathbf{M g}$ & $\mathbf{S}$ \\
\hline 1 & 150 & 95 & 150 & 30 & 30 & 26 \\
\hline 2 & 53 & 50 & 63 & 7 & 1 & 0.7 \\
\hline 3 & 67 & 14 & 69 & 12 & & \\
\hline $\mathbf{4}$ & $\mathbf{5 6}$ & 33 & 31 & & & \\
\hline 5 & 48 & 5 & 90 & 13 & & \\
\hline 6 & 58 & 26 & 48 & & & \\
\hline 7 & 22 & 3 & 14 & 3 & 2 & 1 \\
\hline
\end{tabular}

Fuente: Elaboración propia 
Tabla 7. Rendimiento, peso promedio de fruta de exportación y pachanga en el cultivo de la sandía

\begin{tabular}{|c|c|c|c|}
\hline $\begin{array}{c}\text { Núm. de } \\
\text { parcela }\end{array}$ & $\begin{array}{c}\text { Rendimiento } \\
(\mathbf{K g} / \mathbf{h a})\end{array}$ & $\begin{array}{c}\text { Peso promedio } \\
\text { del fruto }(\mathbf{K g})\end{array}$ & $\begin{array}{r}\text { Pachanga* } \\
(\% \text { total })\end{array}$ \\
\hline 1 & 45.1 & 9.05 & 3.62 \\
\hline 2 & 36.0 & 9.06 & 5.8 \\
\hline 3 & 32.8 & 8.63 & 10.48 \\
\hline 4 & 46.6 & 8.73 & 24.47 \\
\hline 5 & 18.9 & 8.03 & 5.81 \\
\hline 6 & 27.4 & 8.47 & 7.18 \\
\hline 7 & 25.0 & 7.62 & \multicolumn{2}{|c|}{} \\
\hline & $*$ Fruta no comercial y de consumo local
\end{tabular}

Fuente: Elaboración propia

En cuanto a la metodología DOP, indica que el calcio es el principal elemento que limita la producción en el total de las parcelas (tabla 9). Estos resultados hacen pensar que existía algo no relacionado ni con la cantidad de calcio en el suelo ni con la dosis aplicada de fertilización; y fue así que el orden de requerimiento nutrimental por la DOP está más afín con los promedios de los rangos de suficiencia que se utilicen. En la tabla 5 se observa que todos los valores están por debajo del promedio utilizado de referencia (2.38), y es por eso por lo que el $\mathrm{Ca}$ aparece como el elemento más limitante.

Asimismo, al analizar el elemento $\mathrm{S}$ en la tabla 5, en el orden de requerimiento nutrimental es el que menos limita la producción en el total de las parcelas, pero esto es debido también a que todos los valores obtenidos en el estudio están por arriba del valor promedio de referencia $(0.3)$.

Dado que el fertirriego es la aplicación fraccionada del fertilizante en el agua, esta fue medida en número de riegos y horas de duración durante el riego para cada una de las semanas durante el ciclo de desarrollo del cultivo de la sandía (esta acción fue en cada una de las parcelas). En la siguiente tabla se puede comprobar cómo a la parcela número cinco al principio de la plantación, es decir, en las primeras semanas, le hizo falta agua; de manera similar, la parcela siete tuvo un mal manejo, pues cuando el fruto estaba creciendo no se le proporcionó la cantidad adecuada. Así, pues, aunado a las causas anteriores, el agua influyó para que en estas dos parcelas el rendimiento de fruta fuera menor que las demás (ver tabla 8). 
Tabla 8. Número de riegos por semana y horas, total de riegos y metros cúbicos de agua gastados en el ciclo del cultivo de la sandía en Tolimán, Jalisco

\begin{tabular}{|c|c|c|c|c|c|c|c|}
\hline \multirow{2}{*}{ Semanas } & \multicolumn{7}{|c|}{ Núm. de parcela } \\
\cline { 2 - 8 } & 1 & 2 & 3 & 4 & 5 & 6 & 7 \\
\hline $1 .^{\mathrm{a}}$ & 1 & & 4.5 & 1 & 0.5 & 1 & 6 \\
\hline $2 .^{\mathrm{a}}$ & & & 1 & & 0.5 & & \\
\hline $3 .^{\mathrm{a}}$ & & & & 1 & $0.5-1$ & & \\
\hline $4 .^{\mathrm{a}}$ & & & 1 & 2 & 1 & 1 & \\
\hline $5 .^{\mathrm{a}}$ & 11 & 11 & 1 & 2 & 11 & 1 & 1 \\
\hline $6 .^{\mathrm{a}}$ & 11 & 1 & 1 & $4 \mathrm{R}: 1.5$ & 11 & 1 & \\
\hline $7 .^{\mathrm{a}}$ & $4 \mathrm{R}: 1$ & 1 & 2 & $4 \mathrm{R}: 1.5$ & $4 \mathrm{R}: 1$ & 11 & 1 \\
\hline $8 .^{\mathrm{a}}$ & $4 \mathrm{R}: 1$ & 1 & 1.5 & & 11 & 1 & 1 \\
\hline $9 .^{\mathrm{a}}$ & $4 \mathrm{R}: 1$ & 111 & 2 & $7 \mathrm{R}: 1.5$ & 11 & $3 \mathrm{R}: 1.5$ & 1 \\
\hline $10 .^{\mathrm{a}}$ & $7 \mathrm{R}: 1$ & 22 & 11 & $7 \mathrm{R}: 1.5$ & $4 \mathrm{R}: 2$ & $3 \mathrm{R}: 1.5$ & 11 \\
\hline $11 .^{\mathrm{a}}$ & $7 \mathrm{R}: 1$ & $5 \mathrm{R}: 1.5$ & $4 \mathrm{R}: 2$ & $7 \mathrm{R}: 2$ & $6 \mathrm{R}: 1.5$ & $7 \mathrm{R}: 2$ & 11 \\
\hline $1 .^{\mathrm{a}}$ & $7 \mathrm{R}: 1$ & $7 \mathrm{R}: 1.5$ & $7 \mathrm{R}: 2$ & $7 \mathrm{R}: 2.5$ & $7 \mathrm{R}: 2$ & $7 \mathrm{R}: 3$ & $5 \mathrm{R}: 1.5$ \\
\hline $13 .^{\mathrm{a}}$ & $7 \mathrm{R}: 1$ & $7 \mathrm{R}: 2.5$ & $7 \mathrm{R}: 2.5$ & $7 \mathrm{R}: 2.5$ & $7 \mathrm{R}: 1.5$ & $7 \mathrm{R}: 3$ & $6 \mathrm{R}: 2$ \\
\hline $14 .^{\mathrm{a}}$ & $7 \mathrm{R}: 1$ & $6 \mathrm{R}: 2.5$ & $7 \mathrm{R}: 2.5$ & & $7 \mathrm{R}: 2$ & & $7 \mathrm{R}: 2.5$ \\
\hline Total (hrs) & 51 & 52.5 & 73 & 88 & 57 & 72 & 51 \\
\hline M3/ciclo & 1104.9 & 1137.4 & 1581.5 & 1906.5 & 1234.9 & 1559.8 & 1104.9 \\
\hline
\end{tabular}

Fuente: Elaboración propia

\section{Conclusiones y recomendaciones}

Bajo éste estudio y condiciones, se considera que la metodología del Índice de balance de Kenworthy (IBK), es útil para los casos de muestras pequeñas y en estudios muy locales (estudios en valles completos). El trabajo actual es solo un estudio preliminar y se sugiere que el número de observaciones sea cuando menos de 48, porque así se tendrá tres rangos nutrimentales: alto, medio y bajo, donde se repartirían las observaciones en una población normal: 48 / 3 = 16 observaciones por rango, lo que es suficiente para de ahí determinar el rango de suficiencia con valores reales, sin tener que hacer uso de la desviación estándar. Asimismo la determinación de la DOP es una metodología que podría ser utilizada para evaluar el estado nutrimental de diferentes regiones y con observaciones altas y para que en el orden de requerimiento nutrimental sea reflejado realmente el estado nutrimental del estudio en cuestión. Resulta claro que en este estudio la parcela demostrativa no se debió de 
haber fertilizado con magnesio por la cantidad excesiva de este nutriente que el agua de riego contenía. El orden de requerimiento nutrimental nos indica que para obtener altos rendimientos se debe de aumentar la dosis de $\mathrm{S}$.

Con base en los resultados de producción en las parcelas, la dosis aplicada al suelo y el orden de requerimiento nutrimental, se sugiere disminuir la dosis de $\mathrm{P}$ a $60 \mathrm{~kg} / \mathrm{ha}$. Además y de acuerdo a los mismos resultados, la fórmula de fertilización para este valle se sugiere que se aplique de la siguiente manera:

$\begin{array}{llrlll}\mathrm{N} & \mathrm{P} & \mathrm{K} & \mathrm{Ca} & \mathrm{Mg} & \mathrm{S} \\ 150 & 60 & 150 & 30 & 00 & 40\end{array}$

De acuerdo con el IBK, se proponen los siguientes rangos foliares:

$\begin{array}{lccccc}\mathrm{N} & \mathrm{P} & \mathrm{K} & \mathrm{Ca} & \mathrm{Mg} & \mathrm{S} \\ 3.8-3.9 & 0.38-0.41 & 4.1-4.3 & 1.78-2.02 & 0.67-0.68 & 0.53-0.58\end{array}$

\section{Agradecimientos}

No queda más que agradecer a todas aquellas personas que contribuyeron a que este trabajo se llevara a un buen final y como parte del apoyo a los productores de sandía del municipio de Tolimán y su zona de influencia. 


\section{Referencias}

Ayers, R. S. and Westcot, D. W. (1994). Water Quality for Agriculture. Irrigation and Drainage. Rome, Italy: Food and Agriculture Organization of the United Nations Rome.

Bangroo, S. A., Bhat, M. I., Thair, A., Aziz, M. A., Bhat, M. A. and Wani, A. M. (2010). Diagnosis and recommendation Integrated System (DRIS) - A review. International Journal of Current Research, 10, 84-97.

Cadahia, L. C. (2005). Fertirrigación: cultivos hortícolas y ornamentales (3. a ed.). Madrid, España: Ediciones Mundi-Prensa.

Campbell, C. R. and Plank, C. O. (2000). Reference sufficiency ranges for plant analysis in the southern region of the United States. North Carolina, United States: Southern Region Agricultural Experiment Station.

Castellanos, J. Z., Uvalle, B. J. X. y Aguilar, S. A. (2000). Manual de interpretación de análisis de suelos y aguas. México: Intagri.

Fassbender, W. H. y Bornemisza, E. (1987). Química de Suelos. San José, Costa Rica: IICA. Foth, H.D. (1990). Fundamentals of soil science. New York: Wiley

Fuentes, Y. J. L. (2003). Técnica de riego. España: Ed. Mundi-Prensa.

García, E. (1988). Modificaciones al sistema de clasificación climática de Koppen. Ciudad de México, México: Offset Larios.

Howeler, Reinhardt H. (1993). Análisis del tejido vegetal en el diagnóstico de problemas nutricionales: algunos cultivos tropicales. Centro Internacional de Agricultura Tropical, Cali. Colombia. 28 p.

Instituto Nacional de Estadística y Geografía [Inegi]. (1976). Carta edafológica. Cetenal: Venustiano Carranza E-13-B-24. México: Instituto Nacional de Estadística y Geografía.

Instituto Nacional de Estadística y Geografía [Inegi]. (1981). Síntesis de Información Geográfica del Estado de Jalisco. México: Instituto Nacional de Estadística, Geografía e Informática

Instituto Nacional de Estadística y Geografía [Inegi]. (2015). XIII Censo de Población y Vivienda 2010. México: Instituto Nacional de Estadística, Geografía e Informática.

Lamm, R. F., Ayars, E. J. and Nakayama, S. F. (2007). Microirrigation for crop production: design, operation, and management. Oxford, United Kingdom: Elsevier. 
Landriscini, M. R. y Galantini, J. A. (2018). El sistema integrado de diagnóstico y recomendación (DRIS) para conocer el balance nutricional del trigo. En Galantini, J. A. (ed.), Siembra directa en el SO Bonaerense (pp. 13-20). Buenos Aires, Argentina: Universidad Nacional del Sur.

Maldonado, T. R., Etchevers, J. D., Alcántar, G., Rodríguez, J. y Colinas, M. T. (2001). Estado nutrimental del limón mexicano en suelos calcimórficos. Terra latinoamericana, 19(2), 163-174.

Maldonado T. R.; V.G. Almaguer; S.M.E. Álvarez y S.E. Robledo (2008). Diagnóstico nutrimental y validación de dosis de fertilización para limón persa. Terra Latinoamericana. 26(4). pp. 341- 349.

Marín, M.; Pérez de Roberti, R. (1992). Importancia del análisis foliar en la evaluación de la fertilidad de suelos en Venezuela. Una revisión. Fertilidad de Suelos del Postgrado Ciencia del Suelo, Facultad de Agronomía, U.C.V. Revista de Agronomía (LUZ): 9:115. 1992.

Martínez, P. R y Soriano, A. R. (2014). Muestreo para análisis foliar. México: Instituto Nacional de Investigaciones Forestales y Pecuarias (INIFAP). Centro de Investigación Regional Pacífico Centro. Campo Experimental Tecomán.

Montañés, L., Sanz, M. Gómez, V. y Heras, L. (1990). Óptimos nutricionales en melocotonero. An. Aula Dei, 20(1-2), 7-13.

Montañés L., Monge, E., Val, J. and Sanz, M. (1993). Interpretative possibilities of plant analysis by the DOP index. Acta Horticulture (ISHS), (383), 165-190.

Organización de las Naciones Unidas para la Alimentación y la Agricultura [FAO]. (2006). Base Referencial Mundial del Recurso Suelo. Roma, Italia: Organización de las Naciones Unidas para la Alimentación y la Agricultura.

Organización de las Naciones Unidas para la Alimentación y la Agricultura [FAO]. (2008). FAOSTAT. Recuperado de http://faostat.fao.org/site/567/DesktopDefault.aspx?PageID=567\#ancor.

Reis Junior R. dos A. and Monnerat, P. H. (2003). DRIS Norms Validation for Sugarcane Crop. Pesq. Agropec. Bras., 38(3), 1-8.

Richards, L. A. (1977). Diagnóstico y rehabilitación de suelos salinos y sódicos. México: Limusa.

Rincón, S. L. (2005). La fertilización de la lechuga Iceberg. España: Ed. Mundi-Prensa. 
Salazar, G. S. y Lazcano, F. I. (1999). Diagnóstico nutrimental del aguacate "Hass" bajo condiciones de temporal. Revista Chapingo (5), 173-184.

Schulte, E. E. and Kelling, K.A. (2010). Plant analysis: A diagnostic tool. United States: $\begin{array}{llll}\text { Purdue University from } & \text { Retrieved }\end{array}$ http://www.ces.purdue.edu/extmedia/NCH/NCH.46.html.

Secretaría de Agricultura y Desarrollo Rural [Sagarpa]-Servicio de Información Agroalimentaria y Pesquera [SIAP]. (2016). Alcanzan exportaciones de melón, sandía y papaya 283 millones de dólares. México: Secretaría de Agricultura, Ganadería, Desarrollo Rural, Pesca y Alimentación.

Secretaría de Medio Ambiente, Recursos Naturales y Pesca. (1996). NOM-001-ECOL. México: Secretaría de Medio Ambiente, Recursos y Pesca.

Sepúlveda, T. J. L. (2009). Informe de investigación "Estudio de la problemática del bajo rendimiento en el cultivo de la sandía en el Municipio de Tolimán, Jalisco”. Jalisco, México: H. Ayuntamiento de Tolimán.

Servicio de Información Agroalimentaria y Pesquera [SIAP]. (2018). Resumen nacional. Intención de siembra 2018. Ciclo Otoño-invierno. México: Servicio de Información Agroalimentaria y Pesquera.

Thompson, L. M. (1974). El suelo y su fertilidad. Barcelona, España: Reverté.

Troeh, R. F. and Thompson, L. M. (2005). Soils and soil fertility. Victoria, Australia: Blackwell Publishing Asia.

Ventura, U., Salgado, F. S., Castelán, M., Palma, D., Rivera, M. del C. y Sánchez, P. (2012). Métodos de interpretación del análisis nutrimental en naranja valencia (Citrus sinensis) L. Osbeck). Terra Latinoamericana, (30). Recuperado de http://www.redalyc.org/articulo.oa?id=57324446005> . 


\begin{tabular}{|l|l|}
\hline Rol de Contribución & Autor (es) \\
\hline Conceptualización & José de Jesús Sandoval Legazpi. \\
\hline Metodología & $\begin{array}{l}\text { José de Jesús Sandoval Legazpi (Principal); Oswaldo E. } \\
\text { Galindo Verdugo (Igual) }\end{array}$ \\
\hline Software & NO APLICA \\
\hline Validación & NO APLICA \\
\hline Análisis Formal & $\begin{array}{l}\text { José de Jesús Sandoval Legazpi (Principal); Oswaldo E. } \\
\text { Galindo Verdugo (Igual) }\end{array}$ \\
\hline Investigación & $\begin{array}{l}\text { Oswaldo E. Galindo Verdugo (Principal); José de Jesús } \\
\text { Sandoval Legazpi (Igual). }\end{array}$ \\
\hline Recursos & $\begin{array}{l}\text { Araceli de Jesús Arellano Panduro (Apoyo); Lucio Ancira } \\
\text { Sánchez (Apoyo). }\end{array}$ \\
\hline Curación de datos & $\begin{array}{l}\text { José de Jesús Sandoval Legazpi (Principal); Oswaldo E. } \\
\text { Galindo Verdugo (Igual). }\end{array}$ \\
\hline $\begin{array}{l}\text { Escritura - Preparación del } \\
\text { borrador original }\end{array}$ & Oswaldo E. Galindo Verdugo \\
\hline $\begin{array}{l}\text { Escritura - Revisión y } \\
\text { Pdición }\end{array}$ & $\begin{array}{l}\text { José de Jesús Sandoval Legazpi (Principal); Oswaldo E. } \\
\text { Galindo Verdugo (Igual); Araceli de Jesús Arellano Panduro } \\
\text { apoyo); Lucio Ancira Sánchez (Apoyo). }\end{array}$ \\
\hline Supervisión & Oswaldo E. Galindo Verdugo \\
\hline
\end{tabular}

